\title{
Biodiversity of beet webworm microsporidia in Eurasia
}

\author{
Julia Malysh ${ }^{1, *}$, Yuri Tokarev ${ }^{1}$, Svetlana Malysh ${ }^{1}$, Jiang Xingfu $^{2}$, and Andrei Frolov ${ }^{1}$ \\ ${ }^{1}$ All-Russian Institute of Plant Protection, sh. Podbelskogo 3, St. Petersburg, Pushkin 196608 Russia \\ ${ }^{2}$ State Key Laboratory for Biology of Plant Diseases and Insect Pests, Institute of Plant Protection, \\ Chinese Academy of Agricultural Sciences, Beijing, P. R. China
}

\begin{abstract}
The beet webworm Loxostege sticticalis (LS) has eruptive type of population dynamics and high migratory activity. The LS outbreaks are therefore difficult to predict and the pest belongs to the category of highly dangerous pests. However, during periods of depressions this insect is not observed within the most of its range and is very susceptible to infection by pathogens, including various species of microsporidia, some of which are not specific parasites of the order Lepidoptera. The distribution of LS microsporidia in Eurasia is quite extensive. During the study period of LS from 2003 to 2019, we have found 6 species of microsporidia. The parasite list includes not only species known for Lepidoptera such as Nosema sp. and Vairimorpha thomsoni, but also Vairimorpha (Nosema) ceranae as the typical pathogen from Apis mellifera, as well as Endoreticulatus cf poecilimonae, a pathogen similar to Endoreticulatus poecilimonae from Poecilimon thoracicus. Moreover, two isolates from the genus Tubulinosema identified in LS belong to the group of parasites with a very wide host range, including humans. In laboratory experiments, LS proved high sensitivity to microsporidia $N$. pyrausta from Ostrinia nubilalis and N. tyriae from Tyria jacobaeae. Its susceptibility to Paranosema locustae from Locusta migratoria has also been discovered.
\end{abstract}

Beet webworm Loxostege sticticalis (L.) is a dangerous insect pest that damages many agricultural crops such as beets, soybeans, sunflowers, corn and others. It has eruptive type of population dynamics and high migratory activity: adult moths are capable of flying over distances of more than a thousand kilometers. The beet webworm outbreaks are therefore difficult to predict and the pest belongs to the category of highly dangerous pests. At the same time, during periods of depressions this insect is not observed within the most of its range and is very susceptible to infection by pathogens, including various species of microsporidia, some of which are not specific parasites of the order Lepidoptera. The distribution of beet webworms microsporidia in Eurasia is quite extensive (Fig. 1). Beet webworm microsporidia were found in Russia (The Republic of Buryatia, Irkutsk region, Novosibirsk region, Republic of Bashkortostan, Saratov region, Stavropol Territory,

\footnotetext{
*Corresponding author: malyshjm@mail.ru
} 
Krasnodar Territory, Rostov region) and Moldova. A single record is also attributed to a moth sampling from Hebei, China, but without specimen deposition to our collection.

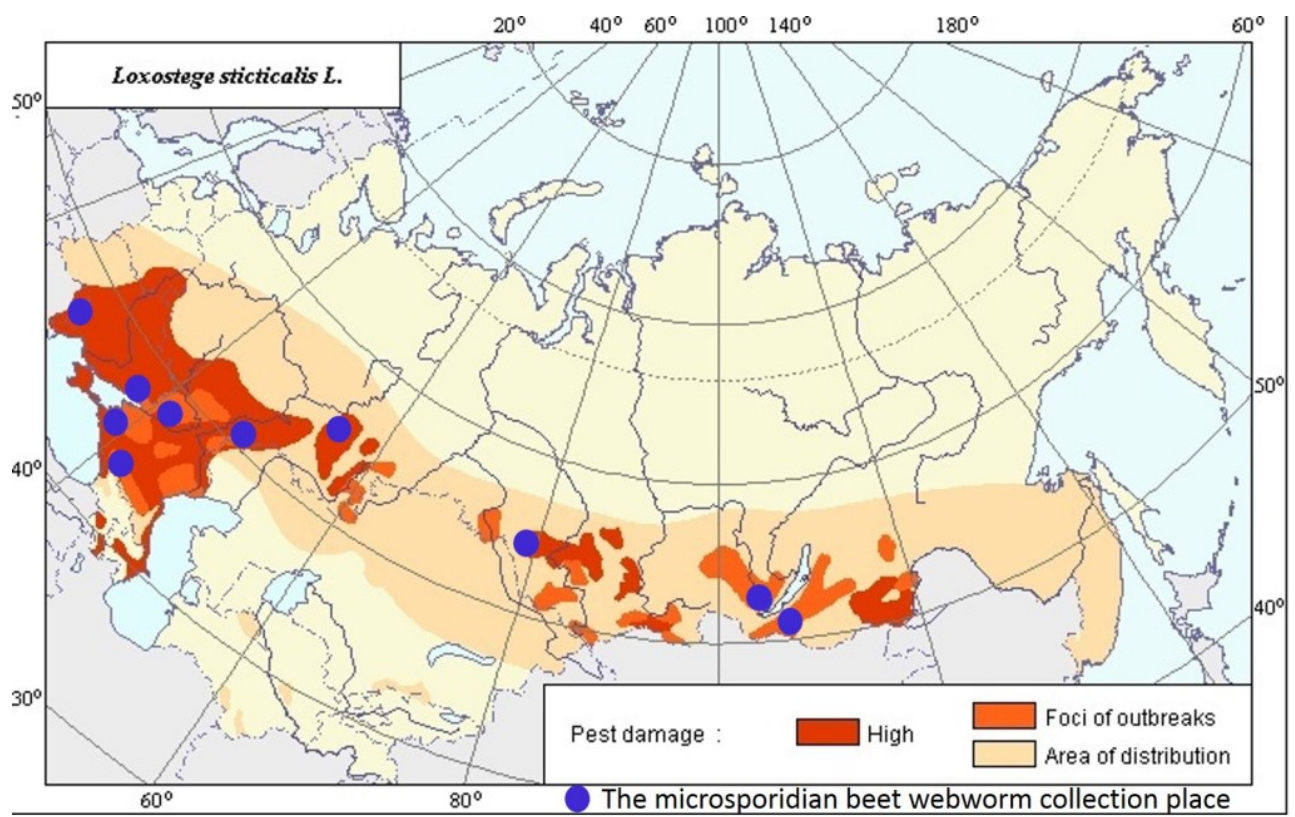

Fig. 1. Microsporidian beet webworm collection places on the map "Area of distribution and harmfulness of the Beet Webworm (Loxostege sticticalis L.)" [1]

Biodiversity of beet webworm microsporidia is presented in Table 1. In the 70-80$\mathrm{s}$ of the 20th century, two species of microsporidia were discovered in beet webworm, namely Nosema loxostegi [2] and Vairimorpha (Nosema) thomsoni [3]. During the study period of the beet webworm from 2003 to 2019 we have found 6 species of microsporidia. The parasite list includes not only species known for Lepidoptera, but also Vairimorpha $N$. ceranae) (according Tokarev et al. (2019) which is the typical pathogen of the honey bee Apis mellifera (L.) (Hymenoptera: Apidae) [4], as well as Endoreticulatus cf poecilimonae [5], a pathogen similar to Endoreticulatus poecilimonae from grasshopper Poecilimon thoracicus (Fieber) (Orthoptera: Tettigoniidae). Moreover, two isolates of the genus Tubulinosema which belong to the group of parasites (family Tubulinosematidae) with a very wide host range, including humans, were identified in the beet webworm [6].

In laboratory experiments, the beet webworm proved high sensitivity to microsporidia $N$. pyrausta from the corn borer Ostrinia nubilalis (Hübner) (Lepidoptera: Pyralidae) and $N$. tyriae from the cinnabar moth Tyria jacobaeae (L.) (Lepidoptera: Arctiidae). Its susceptibility to Paranosema locustae from migratory locusts Locusta migratoria (L.) (Orthoptera: Acrididae) has also been discovered.

Such a high diversity of microsporidia has not been detected in other examined species of lepidopteran insects. For example, only one species of microsporidia, $N$. pyrausta, is characteristic of the corn borer in Eurasia [7]. Three species of microsporidia are known for the corn earworm Helicoverpa armigera (Hübner) and four species for the turnip moth Agrotis segetum (Denis and Schiffermüller) [8].

Our further task is to study the biodiversity of insects that live in the same localities as the beet webworm as possible carriers of microsporidia and other obligate intracellular pathogens. This will provide new knowledge on the role of intracellular pathogens in the phenomenon of outbreak periodicity of the beet webworm. 
Table 1. Prevalence rates of Microsporidia in Loxostege sticticalis sampled across Russia, and adjacent territories

\begin{tabular}{|c|c|c|c|c|c|c|}
\hline \multirow[t]{2}{*}{ \# } & \multirow[t]{2}{*}{$\begin{array}{l}\text { Sampling site, } \\
\text { year, collector }\end{array}$} & \multirow[t]{2}{*}{$\begin{array}{l}\text { Geo- } \\
\text { location }\end{array}$} & \multirow{2}{*}{$\begin{array}{l}\text { Number } \\
\text { of } \\
\text { analyzed } \\
\text { samples } \\
\text { (N) }\end{array}$} & \multirow[t]{2}{*}{$\begin{array}{l}\text { Species of } \\
\text { Microsporidia }\end{array}$} & \multicolumn{2}{|c|}{$\begin{array}{l}\text { Microsporidia prevalence } \\
\text { rates }\end{array}$} \\
\hline & & & & & $\begin{array}{l}\text { Number } \\
\text { of positive } \\
\text { samples, } n\end{array}$ & $\begin{array}{l}\text { Prevalence, } \\
\%(\mathrm{n} / \mathrm{N})\end{array}$ \\
\hline 1 & Bashkiria, 1977 & & \multirow[t]{3}{*}{462} & \multirow{3}{*}{$\begin{array}{l}\text { Nosema } \\
\text { loxostegi sp. n. }\end{array}$} & \multirow[t]{3}{*}{$?$} & \multirow[t]{3}{*}{$3-4$} \\
\hline 2 & Moldova, 1975 & & & & & \\
\hline 3 & $\begin{array}{l}\text { Stavropol } \\
\text { Territory, } 1977\end{array}$ & & & & & \\
\hline 4 & Irkutsk, 1982 & & ? & $\begin{array}{l}\text { Vairimorpha } \\
\text { (Nosema) } \\
\text { thomsoni }\end{array}$ & ? & ? \\
\hline 5 & $\begin{array}{l}\text { Krasnodar } \\
\text { Territory, 2007, } \\
\text { Malysh J.M. }\end{array}$ & $\begin{array}{l}45^{\circ} 40^{\prime} \mathrm{N} \\
37^{\circ} 48^{\prime} \mathrm{E}\end{array}$ & 25 & $\begin{array}{l}\text { Endoreticulatus } \\
\text { sp. }\end{array}$ & 2 & 8 \\
\hline 6 & $\begin{array}{ll}\text { Republic } & \text { of } \\
\text { Bashkortostan, } \\
2008\end{array}$ & & 36 & $?$ & 2 & 5.6 \\
\hline 7 & $\begin{array}{lr}\text { Salsk, } & \text { Rostov } \\
\text { region, } & 2005, \\
\text { Malysh } & \text { J.M., } \\
\text { Tokarev } & \text { Y.S. } \\
\end{array}$ & $\begin{array}{l}46^{\circ} 30^{\prime} \mathrm{N} \\
41^{\circ} 19^{\prime} \mathrm{E}\end{array}$ & 28 & ? & 2 & 7 \\
\hline 8 & $\begin{array}{l}\text { Chertkovsk } \\
\text { District, Rostov } \\
\text { region, 2013, } \\
\text { Artokhin K.S. }\end{array}$ & $\begin{array}{l}49^{\circ} 16^{\prime} \mathrm{N} \\
40^{\circ} 38^{\prime} \mathrm{E}\end{array}$ & 100 & - & 0 & 0 \\
\hline \multirow[t]{3}{*}{9} & \multirow{3}{*}{$\begin{array}{l}\text { Neklinovsk } \\
\text { District, Rostov } \\
\text { region, 2013, } \\
\text { Artokhin K.S. }\end{array}$} & \multirow[t]{3}{*}{$\begin{array}{l}47^{\circ} 16^{\prime} \mathrm{N} \\
38^{\circ} 37^{\prime} \mathrm{E}\end{array}$} & \multirow[t]{3}{*}{100} & $\begin{array}{l}\text { Vairimoroha } \\
\text { (Nosema) } \\
\text { ceranae }\end{array}$ & 3 & 3 \\
\hline & & & & Nosema sp. & 4 & 4 \\
\hline & & & & $\begin{array}{l}\text { Tubulinosema } \\
\text { sp. }\end{array}$ & 35 & 35 \\
\hline 10 & $\begin{array}{l}\text { Novosibirsk } \\
\text { region, 2010, } \\
\text { Tokarev Y.S. }\end{array}$ & $\begin{array}{l}53^{\circ} 42^{\prime} \mathrm{N} \\
77^{\circ} 45^{\prime} \mathrm{E}\end{array}$ & 50 & $\begin{array}{l}\text { Tubulinosema } \\
\text { loxostegi }\end{array}$ & 12 & 24 \\
\hline 11 & $\begin{array}{l}\text { Saratov Region, } \\
\text { 2006, Silaev A.I. }\end{array}$ & $\begin{array}{l}51^{\circ} 27^{\prime} \mathrm{N} \\
46^{\circ} 12^{\prime} \mathrm{E}\end{array}$ & 30 & $?$ & 13 & 43 \\
\hline 12 & $\begin{array}{l}\text { Saratov Region, } \\
\text { 2013, Silaev A.I. }\end{array}$ & $\begin{array}{l}51^{\circ} 27^{\prime} \mathrm{N} \\
46^{\circ} 12^{\prime} \mathrm{E}\end{array}$ & 30 & $?$ & 1 & 3 \\
\hline 13 & $\begin{array}{l}\text { Irkutsk Region, } \\
2010 \text {, Belyakova } \\
\text { N.A. }\end{array}$ & $\begin{array}{l}52^{\circ} 16^{\prime} \mathrm{N} \\
104^{\circ} 19^{\prime} \mathrm{E}\end{array}$ & 30 & $\begin{array}{l}\text { Vairimorpha } \\
\text { (Nosema) } \\
\text { thomsoni }\end{array}$ & 23 & 77 \\
\hline 14 & $\begin{array}{l}\text { Buryatia, 2009, } \\
\text { Akhanaev Y.B. }\end{array}$ & $\begin{array}{l}52^{\circ} 09^{\prime} \mathrm{N} \\
106^{\circ} 36^{\prime} \mathrm{E}\end{array}$ & 30 & $\begin{array}{l}\text { Vairimorpha } \\
\text { (Nosema) } \\
\text { thomsoni }\end{array}$ & 10 & 33 \\
\hline
\end{tabular}




\section{References}

1. M.A. Chumakov, T.L. Kuznetsova, M.I. Saulich, Agroatlas (2004) http://www.agroatlas.ru/en/content/pests/Loxostege_sticticalis/map/

2. I.V. Issi, P.M. Simchuk, D.F. Radishcheva, Bulleten VIZR, 48 (1980)

3. J.M. Malysh, A.G. Kononchuk, A.A. Nurzhanov, A.N. Frolov, I.V. Issi, Y.S. Tokarev, Euroasian. J. Entomol., 12, 6 (2013)

4. J.M. Malysh, A.N. Ignatieva, K.S. Artokhin, A.N. Frolov, Y.S. Tokarev, Parasitol. Res., 117, 9 (2018) http://doi.org/10.1007/s00436-018-5987-3

5. J.M. Malysh, A.G. Kononchuk, A.N. Frolov, Plant Protection News [Vestnik zashchity rasteniy] 2(100), 45-51 (2019) http://doi.org/10.31993/2308-6459-2019-2(100)-45-51

6. J.M. Malysh, Y.S. Tokarev, A.N. Frolov, I.V. Issi, N.V. Sitnicova, V.V. Martemyanov, Acta Protozool., 52, 4 (2013) http://doi.org/10.4467/16890027AP.13.027.1319

7. I.V. Grushevaya, A.N. Ignatieva, S.M. Malysh, I.V. Senderskiy, I.V. Zubarev, A.G. Kononchuk, Acta $\quad$ Protozool., $\quad 57 \quad$ (2018) http://doi.org/10.4467/16890027AP.18.004.8398

8. I.V. Issi, G.N. Nilova, Izvest. Akad. Nauk Tadzhiksk. SSR, 1, 26 (1967) 